\title{
DATA FOR WEAK LINES
}

\author{
A. HIBBERT \\ Department of Applied Mathematics and Theoretical Physics, \\ The Queen's University of Belfast, Belfast BT7 1NN, N. Ireland
}

Weak lines, being unsaturated, are particularly important for the determination of interstellar abundances of elements. From a theoretical point of view, there are several possible causes of an electric dipole transition having a small oscillator strength. In some cases, several contributions to the dipole matrix element substantially cancel (e.g. the $1808 \AA$ line Si II), while in other cases, the oscillator strength would be zero but for the inclusion of small relativistic effects (e.g. the intersystem $1909 \AA$ line in C II).

For weak lines, the interstellar abundances depend on the oscillator strength (calculated or measured) and the equivalent width (observed). The increased resolution of the Goddard High Resolution Spectrograph on board the Hubble Space Telescope provides for many lines equivalent widths of much greater precision than was previously available. This situation presents a challenge to theorists to improve the quality of their calculations of oscillator strengths.

Such calculations are difficult for weak transitions, especially when the cause is cancellation. Of course, with larger and more powerful computers available, it is possible to undertake more extensive calculations. But the quality of observational data now makes it imperative for theorists not merely to do a better calculation than before, but to give a considered estimate of the accuracy of the results. Since there are no effective theoretical methods for obtaining rigorous bounds to oscillator strengths which could give a useful guide to accuracy, theoretical estimates of accuracy can be achieved only by undertaking a systematic sequence of calculations of increasing complexity, while ensuring that no significant contributions to the calculations are omitted.

It is only recently that such a systematic approach has been feasible. Prior to that, theoretical values of small oscillator strengths have widely differed - up to factors of ten or so. This has encouraged the practice of using "accepted" abundances along with observed equivalent widths to deduce values for oscillator strengths of some lines. Sometimes this has worked well. Certainly it is a useful guide for theorists. But there are other instances where the ensuing oscillator strengths differ markedly from theoretical calculations, even for more normal allowed transitions where no significant difficulties arise in the calculations. The problem for the interaction between atomic physicists and astrophysicists is that these observationally deduced oscillator strengths have sometimes been accepted by astrophysicists as definitive, when clearly they are not. The only satisfactory way of clarifying this situation is to undertake a systematic study of specific weak transitions and to examine the convergence of the oscillator strengths 
as the relevant wave functions are improved.

Laboratory experimental determinations are also important. Weak transitions have been difficult to observe because of their small signals and the long lifetimes of the upper levels of the transitions, but recently new techniques have allowed measurements to be made of reasonable accuracy. What is really significant is that these new measurements agree rather well with the most systematic and accurate calculations.

We give two examples:

The $1808 \AA$ line in Si II corresponds to the $3 s^{2} 3 p^{2} P_{3 / 2}-3 s 3 p^{2}{ }^{2} D_{5 / 2}$ transition. There is strong mixing in the upper state between the ${ }^{2} \mathrm{D}$ configurations $3 s 3 p^{2}$ and $3 s^{2} 3 d$. The mixing results in severe cancellation in the dipole matrix element and leads to an oscillator strength two orders of magnitude smaller than that of the $3 s^{2} 3 p^{2} P_{3 / 2}-3 s^{2} 3 d^{2} D_{3 / 2}$ transition. Early calculations of the oscillator strength ranged from 0.0115 (Nussbaumer (1977)) to 0.001 (Froese Fischer (1981), Dufton et al (1983)), while an observationally derived value of 0.0055 was obtained by Shull et al (1981) recently, Dufton et al (1992) have undertaken a systematic study of the effect on the oscillator strength of a systematic improvement in the wave functions. They obtain 0.0020 , with an estimated accuracy of $\pm 25 \%$. Bergeson and Lawler (1993), using a time-resolved LIF technique to measure this oscillator strength obtain the value 0.0021 with an estimated error of $10 \%$. The agreement between theory and experiment is remarkable, in view of the difficulties encountered in each approach. Spitzer and Fitzpatrick (1993) have shown that these new, essentially coinciding values are entirely consistent with oscillator strengths of other allowed Si II transitions in their analysis of observational data.

The $1909 \AA$ transition in C III corresponds to $2 s^{2}{ }^{1} S_{0}-2 s 2 p^{3} P_{l}^{\circ}$. Intersystem lines of light elements should be fairly simple to calculate, but again a range of oscillator strength values appears in the literature. Accuracy of calculations can be assessed by comparing energy differences between relevant levels with corresponding experimental values. The inaccuracy in the calculation of these quantities enables an estimate of the level of accuracy of the oscillator strength to be determined. In two independent calculations, Fleming et al. (1994) obtained for the transition probability $104 \pm 4 \mathrm{~s}^{-1}$ while Froese Fischer (1994) obtained $103 \pm 3$ $\mathrm{s}^{-1}$. The closeness and accuracy of these results suggests that the value of $121 \pm 7 \mathrm{~s}^{-1}$ obtained by Kwong et al (1993) using the ion-trap technique is rather too high.

It is clear that while calculations of rather high accuracy can now be achieved in these difficult cases, considerable effort has to be made. So values for these weak lines, when determined almost incidentally as part of a calculation of many transitions simultaneously, should be treated with caution.

Bergeson, S.D. \& Lawler, J.E., ApJ, 414, L137

Dufton, P.L., Hibbert, A., Kingston, A.E. \& Tully, J.A., 1983, MNRAS, 202, 145

Dufton, P.L., Keenan, F.P., Hibbert, A., Ojha, P.C. \& Stafford, R.P., 1992, ApJ, 387, 414

Fleming, J., Hibbert, A. \& Stafford, R.P., 1994, Phys. Scripta, 49, 316

Froese Fischer, C., 1994, Phys. Scripta, 49, 323 
Kwong, V.H.S., Fang, Z., Gibbons, T.T., Parkinson, W.H. \& Smith, P.L., 1993, ApJ, 411, 431 Nussbaumer, H., 1977, A\&A, 58, 291

Shull, J.M., Snow, T.P. \& York, D.G., 1981, ApJ, 246, 549

Spitzer, L. \& Fitzpatrick, E.L., 1993, ApJ, 409, 299

\section{MOLECULAR DATA FOR STELLAR OPACITIES}

\section{UFFE GRÅE JØRGENSEN}

\section{Niels Bohr Institute, Blegdamsvej 17, DK-2100 Copenhagen, Denmark}

In total, 40 neutral diatomic molecules, 2 molecular ions, and 7 polyatomic molecules are known from observed photospheric stellar spectra. Line data for opacity computations (i.e., lists of line frequencies, intensities, and excitation energies) exist for 17 of these molecules, although the data are complete only for a handful of them. A detailed description of stellar photospheric molecules can be found in Tsuji (1986), and the existing opacity data have been reviewed by Jorgensen (1995).

Listed line frequencies in the data bases are either the measured values, or based on computed molecular constants obtained from fits to measured values. Attempts to compute $a b$ initio line frequencies have so far resulted in lower accuracy than what is obtained by use of molecular constants. Published line strengths include measured values as well as ab initio values. For strong bands the ab initio intensities are as accurate as the laboratory values, whereas measured values for weak bands are generally more accurate than the ab initio values. The primary advantage of ab initio computations is therefore that the complete set of all transitions can be obtained. Exploratory studies have shown that completeness of the line data is crucial for the obtained stellar photospheric structure.

As an alternative to the ab initio computations of the line intensities, fits to experimental data have been attempted. The most promising method seems to be to fit the dipole function by use of a Padé approximant. Combined with a potential fitted to experimental energy levels, such a dipole function can in principle be used to predict the complete list of band intensities and line intensities for all bands with energies up to the molecular dissociation energy. The part of the dipole function which corresponds to the largest stretching (or bending) of the molecule is the most uncertain in such fits as well as in ab initio computations. This part is responsible for most of the many weak transitions, and large uncertainties are therefore to be excepted in the computed intensities of the weak spectral bands. As these are of major importance for the stellar photospheric structure (due to their huge number and their pseudo continuous appearance in the spectrum), a particularly large effort is desirable in comparing computed intensities with laboratory data for a representative sample of weak bands. Unfortunately, only few measurements of weak bands exist. Attempts to measure all the existing bands are not recommended, because of 